\title{
MicroRNA-222 Promotes the Proliferation of Pulmonary Arterial Smooth Muscle Cells by Targeting P27 and TIMP3
}

\author{
Ying Xua Yihua Beib Shutong Shen ${ }^{\mathrm{a}}$ Jialiang Zhang ${ }^{\mathrm{a}}$ Yichao Lu $^{\mathrm{a}}$ Junjie Xiao \\ Xinli Lia
}

aDepartment of Cardiology, The First Affiliated Hospital of Nanjing Medical University, Nanjing, ${ }^{\text {bC } C a r d i a c}$ Regeneration and Ageing Lab, School of Life Science, Shanghai University, Shanghai, China

\section{Key Words}

MiR-222 - Pulmonary artery smooth muscle cells - Pulmonary artery hypertension • Proliferation

\begin{abstract}
Background/Aims: Aberrant vascular smooth muscle cell (VSMC) proliferation plays an important role in the development of pulmonary artery hypertension (PAH). Dysregulated microRNAs (miRNAs, miRs) have been implicated in the progression of PAH. miR-222 has a pro-proliferation effect on VSMCs while it has an anti-proliferation effect on vascular endothelial cells (ECs). As the biological function of a single miRNA could be cell-type specific, the role of miR-222 in pulmonary artery smooth muscle cell (PASMC) proliferation is not clear and deserves to be explored. Methods: PASMCs were transfected with miR-222 mimic or inhibitor and PASMC proliferation was determined by Western blot for PCNA, Ki-67 and EdU staining, and cell number counting. The target genes of miR-222 including P27 and TIMP3 were determined by luciferase assay and Western blot. In addition, the functional rescue experiments were performed based on miR-222 inhibitor and siRNAs to target genes. Results: miR-222 mimic promoted PASMC proliferation while miR-222 inhibitor decreased that. TIMP3 was identified to be a direct target gene of miR-222 based on luciferase assay. Meanwhile, P27 and TIMP3 were up-regulated by miR-222 inhibitor and down-regulated by miR-222 mimic. Moreover, P27 siRNA and TIMP3 siRNA could both attenuate the anti-proliferation effect of miR-222 inhibitor in PASMCs, supporting that P27 and TIMP3 are at least partially responsible for the regulatory effect of miR-222 in PASMCs. Conclusion: miR-222 promotes PASMC proliferation at least partially through targeting P27 and TIMP3.
\end{abstract}

C 2017 The Author(s)

Published by S. Karger AG, Basel

\section{Introduction}

Pulmonary arterial hypertension (PAH), a progressive disorder characterized by pulmonary vascular remodeling, can lead to an increase in pulmonary vascular resistance,

Y. Xu and Y. Bei contributed equally to this work. 
right heart failure, and ultimately death $[1,2]$. Excessive pulmonary artery smooth muscle cell (PASMC) proliferation is one of the most prominent features of $\mathrm{PAH}$, which can lead to the narrowing or occlusion of pulmonary vessels and therefore plays an important role in the occurrence and development of PAH [3].

MicroRNAs (miRNAs, miRs) are a class of endogenous, small, non-coding RNAs that can regulate gene expressions by targeting the 3'untranslational regions (UTRs) of mRNAs [4, 5]. Being the central regulators of gene expressions, miRNAs participate in many essential biological processes, such as cell metabolism, development, proliferation, and death [68]. Of note, miRNAs have also been implicated in the development of PAH [9-11]. In fact, multiple miRNAs such as miR-21, -17, -30c, -125a, -126, -143, -145, -206, -130/301, and -328 have been identified to be responsible for vascular remodeling in PAH [12-24]. miR222 has been reported to promote cardiomyocyte hypertrophy and proliferation in exerciseinduced cardiac growth [25]. However, chronic cardiac-specific overexpression of miR-222 might lead to cardiac remodeling and heart failure [26]. miR-222 also plays important roles in multiple cancer types $[27,28]$. Moreover, miR-222 was found to regulate essential pathophysiological vascular processes [29]. For example, miR-222 has a pro-proliferation effect on vascular smooth muscle cells (VSMCs) [30], while it has an anti-proliferation effect on vascular endothelial cells (ECs) [31]. As the biological function of a single miRNA could be cell-type specific, the role of miR-222 in PASMC proliferation is not clear and deserves to be explored.

\section{Materials and Methods}

This study was approved by the ethical committees of the Nanjing Medical University and all animal experiments were conducted under the guidelines on humane use and care of laboratory animals for biomedical research published by National Institutes of Health (No. 85-23, revised 1996).

\section{Cell culture}

The primary PASMCs were isolated from the pulmonary arteries of male Sprague-Dawley rats ( 5 weeks old) by using a tissue-sticking method. After the dissection of adventitia and endothelia, pulmonary arteries were quickly cut into small pieces and stuck to cell culture bottle. The primary PASMCs were confirmed by immunofluorescent stainings for SM $\alpha$-actin ( $\alpha$-SMA) and Desmin (Sigma-Aldrich, St. Louis, MO, USA). The basic culture medium was consisted of DMEM-F12 supplemented with $5 \%$ fetal bovine serum (FBS) while the starvation medium was with 1\% FBS. The primary PASMCs between passage 3 and 6 were used for experiments.

\section{Hypoxia}

Hypoxic condition was created in a hypoxic $\left(1 \% \mathrm{O}_{2}\right.$ and $\left.5 \% \mathrm{CO}_{2}, 37^{\circ} \mathrm{C}\right)$ incubator (Billups-Rothenberg, INC. California, United States). Experiments were performed after 24 hours of hypoxic incubation, at which stage cell lysate and total RNA were collected.

Transfection

Before transfection, PASMCs were starved for 8 hours for cycle synchronization. Lipofectamine 2000 reagent was used to transfect miR-222 mimic (50 nM), miR-222 inhibitor (100 nM), and their negative control (NC) into the different groups according to the instructions. siRNA-P27(Kip1) and/or siRNA-TIMP3 were transfected to PASMCs to knock-down P27 and/or TIMP3. After incubation for 48 hours, the effect of siRNAs was confirmed by real-time polymerase chain reactions (PCRs).

P27 siRNA sequence: sense (5'-3'): GCGGCAGAAGAUUCUUCUUTT, anti-sense (5'-3'): AAGAAGAAUCUUCUGCCGCTT.

TIMP3 siRNA sequence: sense (5'-3'): GCUAUCAGUCCAAACACUATT, anti-sense (5'-3'): UAGUGUUUGGACUGAUAGCTT.

NC siRNA sequence: sense (5'-3'): UUCUCCGAACGUGUCACGUTT, anti-sense (5'-3'): ACGUGACACGUUCGGAGAATT. 


\section{Cellular Physiology Cell Physiol Biochem 2017;43:282-292 \\ \begin{tabular}{l|l|l} 
and Biochemistry $10.1159 / 000480371$ & $\begin{array}{l}\text { C } 2017 \text { The Author(s). Published by S. Karger AG, Basel } \\
\text { www.karger.com/cpb }\end{array}$ \\
\hline
\end{tabular} \\ Xu et al.: MicroRNA-222 Promotes Pulmonary Arterial Smooth Muscle Cells Proliferation}

Quantitative reverse transcription PCRs ( $q R T-P C R s$ )

Briefly, total RNA was extracted from cells with miRNeasy Mini Kit (Qiagen, Hilden, Germany). For the evaluation of miR-222 level, mature miRNA was reverse transcribed with Bulge-Loop ${ }^{\text {TM }}$ miRNA qPCR Primers (Ribobio, Guangzhou, China) prior to qPCR according to the manufacturer's instructions. qRT-PCR for miR-222 cDNA synthesis was performed with Bio-Rad iScript ${ }^{\mathrm{TM}}$ cDNA Synthesis Kit (Bio-Rad, Hercules, CA, USA). For quantitative miRNA analysis, a template equivalent to $400 \mathrm{ng}$ of total RNA was subjected to 40 cycles of quantitative PCR using the Takara SYBR Premix Ex TaqTM (Tli RNaseH Plus, Takara, Tokyo, Japan) in the 7900HT Fast Real-Time PCR System.

\section{Immunofluorescence}

For immunofluorescence, PASMCs were determined by SM $\alpha$-actin ( $\alpha$-SMA) and Desmin. The apoptosis was examined by TdT-mediated dUTP nick end labeling (TUNEL) assay using In Situ Cell Death Detection Kit according to the manufacturer's instructions (Roche, Mannheim, Germany). The proliferation of PASMCs was determined by 5-ethynyl-2'-deoxyuridine (EdU) assay, Ki-67 staining, and cell number counting. For EdU assay, EdU was added to the culture medium for 8 hours in order to incorporate into replicating cells' DNA. Then cultured cells were washed three times with PBS and fixed with 4\% paraformaldehyde for $20 \mathrm{~min}$. $0.2 \%$ Triton X-100 was used to permeabilize the nuclear membrane and PBS containing 10\% goat serum was used for blocking for 1 hour at room temperature. Ultimately, PASMCs were stained by Cell-Light ${ }^{\mathrm{TM}}$ EdU Apollo ${ }^{\circledR} 488$ In Vitro Imaging Kit (Life Technologies, New York, USA) according to the instructions. For Ki-67 staining, after fixation, permeabilization, and blocking, PASMCs were incubated with Ki-67 antibody (1:500 dilution; Abcam, Cambridge, MA, USA) at $4^{\circ} \mathrm{C}$ overnight and then stained with Alexa Fluor 488 goat anti-rabbit IgG antibody for 2 hours at room temperature. For cell number counting, at least 200 cells or 10 images were quantified in each well to get accurate numbers for each group. Nuclei were stained with DAPI. Finally, cells were detected with a fluorescence microscope.

\section{Western blot}

The total protein was extracted from lysed PASMCs. After determined by BCA protein assays, equal quantities of protein were subjected to $10 \%$ sodium dodecyl sulfate-polyacrylamide gel electrophoresis (SDS-PAGE) and transferred onto the PVDF membranes. The membranes were blocked by Tris-buffered saline Tween-20 (TBST) containing 5\% BSA for 2 hours at room temperature and then incubated on a rotary shaker (80 rpm/min) with antibodies against B-cell lymphoma 2 (Bcl-2, 1:1000 dilution; Cell Signaling Technology, Boston, Massachusetts, USA), Bcl-2-associated X protein (Bax, 1:1000 dilution; Cell Signaling Technology, Boston, Massachusetts, USA), Proliferating Cell Nuclear Antigen (PCNA, 1:1000 dilution; Cell Signaling Technology, Boston, Massachusetts, USA), P27 (1:1000 dilution; Abcam, Cambridge, UK), Tissue Inhibitor of Metalloproteinase 3 (TIMP3, 1:1000 dilution, Cell Signaling Technology, Boston, Massachusetts, USA), respectively. $\beta$-actin (1:1000 dilution; Cell Signaling Technology, Boston, Massachusetts, USA) and glyceraldehyde-3-phosphate dehydrogenase (1:1000 dilution; Cell Signaling Technology, Boston, Massachusetts, USA) were used as a loading control. After incubation with the HRP-conjugated rabbit antigoat IgG at room temperature for 1 hour, the protein bands were quantified with ECL Plus Western blotting detection reagents (Bio-Rad) and ChemiDoc XRS Plus luminescent image analyzer (Bio-Rad, Hercules, CA, USA).

\section{Luciferase assay}

A fragment of the 3'-UTR of TIMP3 mRNA containing the putative miR-222 binding sequences was cloned into the firefly luciferase reporter construct pmiR-RB-Report (Ribobio, Guangzhou, China). For the reporter assay, human embryonic kidney 293T cells (HEK293T cells) were seeded onto 96-well plates and co-transfected with TIMP3-3'UTR-Luc $(1 \mu \mathrm{g})$ and miR-222 mimic (50 nM) by Lipofectamine 2000. In addition, the construct with mutated fragment of the 3'-UTR of TIMP3 without putative miR-222 binding sequences was used as a mutated control. Following 48 hour incubation, luciferase activity was measured on a scintillation counter by a dual luciferase reporter system (GeneCopoeia ${ }^{\mathrm{TM}}$ Luc-Pair $^{\mathrm{TM}}$ Duo-Luciferase Assay kit 2.0, USA).

\section{Statistical analysis}

All data were expressed as mean \pm SE. For relative gene expression, proliferation rate, and cell counting, the mean value of negative control group was defined as $100 \%$ or 1.0 . Two group samples were 


\section{Cellular Physiology

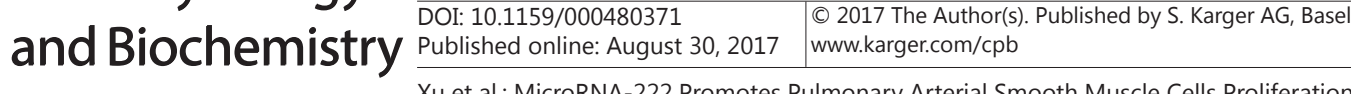

compared by an independent-samples $\mathrm{T}$ test. Three or more group samples were compared by one-way ANOVA followed by Bonferroni's post-hoc test. A $P$ value $<0.05$ was considered to be statistically significant.

\section{Results}

miR-222 promotes PASMC proliferation

The primary PASMCs were specified by immunofluorescent stainings for $\alpha$-SMA and Desmin (Fig. 1A). To explore the effect of miR-222 on PASMC proliferation, three different methods were used including EdU assay, Ki-67 staining, and cell number counting. As shown in Fig. 1B, miR-222 mimic increased whereas miR-222 inhibitor decreased the expression level of miR-222 in cultured PASMCs 48 hours after transfection. In addition, miR-222 mimic up-regulated PCNA expression while miR-222 inhibitor down-regulated PCNA expression (Fig. 1C). The Bax/Bcl2 ratio and PASMC apoptosis were not significantly influenced after transfection with miR-222 mimic compared with the negative control mimic (Fig. 1D and E). In accordance with the Western blot result of PCNA, miR-222 mimic increased the percentage of EdU positive cells, Ki-67 positive cells, and the total number of PASMCs (Fig. 1F-H). In contrast, miR-222 inhibitor decreased PASMC proliferation (Fig. 1F-H). These results suggested that miR-222 had a pro-proliferation effect on PASMCs.

P27 is a target gene of miR-222 in PASMCs

P27 is the most well-known target gene of miR-222 [32,33]. To determine whether P27 was putative target gene of miR-222 in PASMCs, we detected the P27 protein level by Western blot after up- or down-regulation of miR-222. As expected, P27 was up-regulated by miR-222 inhibitor and down-regulated by miR-222 mimic (Fig. 2A). These data indicate that P27 is a target gene of miR-222 in PASMCs.

The effect of P27 siRNA used in the study was confirmed by Western blot (Fig. 2B). We found that P27 knockdown by P27 siRNA could significantly increase the proliferation of PASMCs as determined by Ki-67 staining, EdU staining, and cell number counting, while co-transfection of miR-222 mimic and P27 siRNA did not further increase the proliferation of PASMCs (Fig. 2C-E), indicating that P27 inhibition might mediate the pro-proliferation effect of miR-222 in PASMCs. Besides that, P27 siRNA could attenuate the anti-proliferation effect of miR-222 inhibitor in PASMCs (Fig. 2F-H), supporting that P27 is at least partially responsible for the regulatory effect of miR-222 on PASMC proliferation.

TIMP3 is another target gene of miR-222 in PASMCs

TIMP3 has also been reported to be a target gene of miR-222 in several types of cells $[34,35]$. However, if TIMP3 is a target gene of miR-222 in PASMCs remains undetermined. We cloned the 3'UTR of TIMP3 mRNA containing the putative miR-222 binding sequences into the firefly luciferase reporter construct pmiR-RB-Report. Luciferase assay showed that exogenous miR-222 led to the reduction of luciferase activity in cells transfected with the construct with 3'UTR of TIMP3, but had no effect when the putative miR-222 binding site in TIMP3 was mutated (Fig. 3A and B). This suggests that TIMP3 is a direct target of miR-222. Besides that, we also determined the expression level of TIMP3 in the presence of miR-222 mimic or inhibitor and found that miR-222 could negatively regulate TIMP3 protein level in PASMCs (Fig. 3C).

The effect of TIMP3 siRNA used in this study was confirmed by Western blot (Fig. 4A). TIMP3 knockdown by siRNA could significantly enhance the proliferation of PASMCs as determined by Ki-67 staining, EdU staining, and cell number counting, while co-transfection of miR-222 mimic and TIMP3 siRNA did not further enhance the proliferation of PASMCs (Fig. 4B-D), indicating that TIMP3 inhibition might also be responsible for the pro-proliferation effect of miR-222 in PASMCs. Moreover, TIMP3 siRNA could attenuate the anti-proliferation effect of miR-222 inhibitor in PASMCs (Fig. 4E-G), supporting that TIMP3 at least in part mediates the regulatory effect of miR-222 on PASMC proliferation. 
$\mathbf{A}$

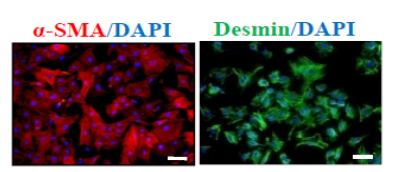

C
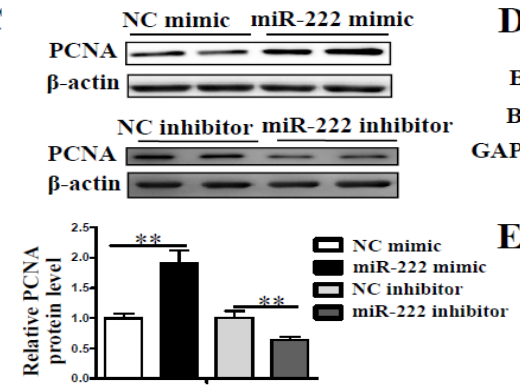

F

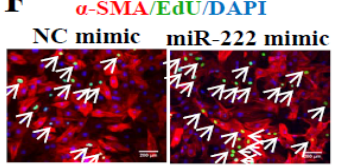

NC inhibitor miR-222 inhibitor

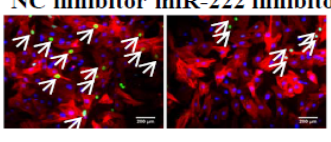

$\mathbf{H}$

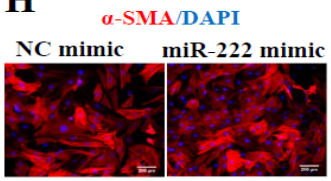

NC inhibitor miR-222 inhibitor

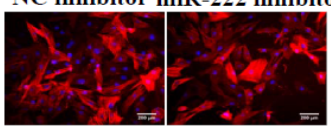

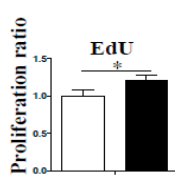
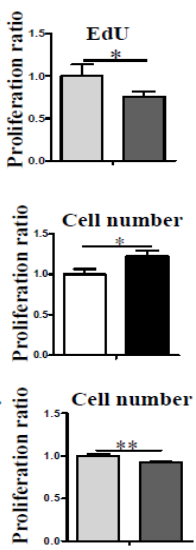

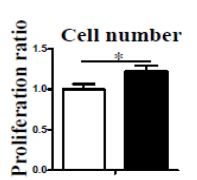

B

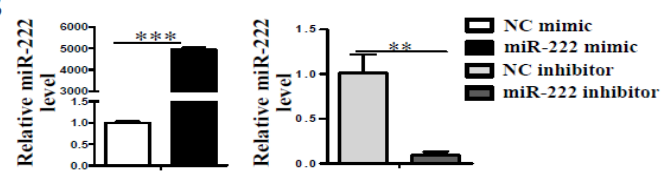

D

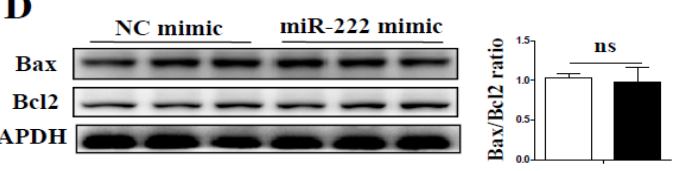

E $\alpha$-SMA/TUNEL/DAPI

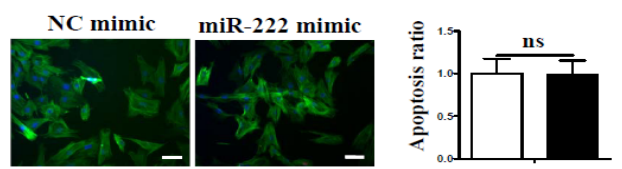

G

a-SMA/Ki67/DAPI

NC mimic miR-222 mimic NC inhibitor miR-222 inhibitor
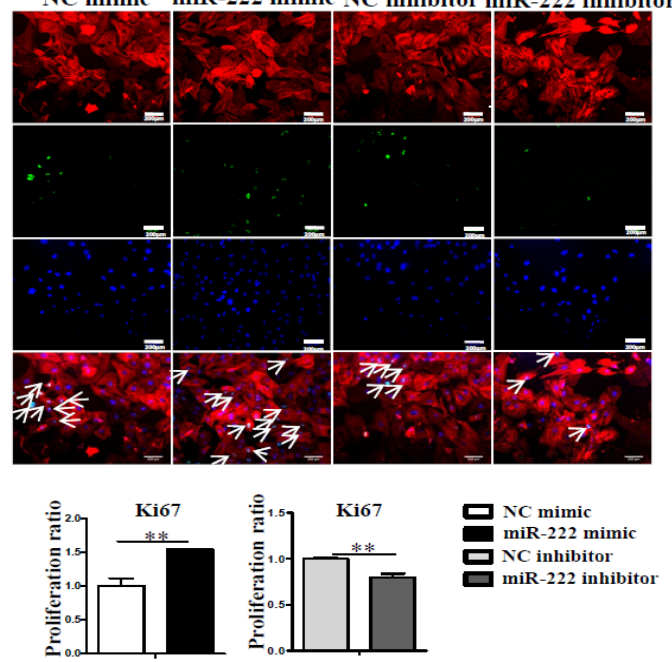

NC inhibitor

miR-222 inhibitor
들 Mimic

Fig. 1. miR-222 promotes pulmonary artery smooth muscle cell (PASMC) proliferation. (A) PASMCs were specified by immunofluorescent stainings for $\alpha$-SMA and Desmin. (B) The relative miR-222 expression level was increased by miR-222 mimic and decreased by miR-222 inhibitor as determined by qRT-PCR. NC mimic and NC inhibitor were used as negative controls. (C) miR-222 mimic increased PCNA expression, while miR222 inhibitor decreased PCNA expression at protein level. (D) The Bax/Bcl2 protein ratio was not changed in PASMCs transfected with miR-222 mimic. (E) TUNEL assay showed that the apoptosis of PASMCs was not changed after transfection with miR-222 mimic. (F-H) miR-222 mimic promoted, while miR-222 inhibitor decreased PASMC proliferation as determined by EdU assay (F), Ki67 staining (G), and quantification of cell number $(\mathrm{H})$. For immunofluorescent stainings, nuclei were stained by DAPI. The arrows indicate EdUpositive or Ki67-positive PASMCs. Scale bar: $200 \mu \mathrm{m}$. ${ }^{*}, P<0.05$; **,$P<0.01$; ${ }^{* * *}, P<0.001 . \mathrm{n}=6$ per group.

To further clarify if concomitant silencing of P27 and TIMP3 has additive effect on PASMC proliferation, the P27 and TIMP3 were simultaneously knocked-down by co-transfection of P27 siRNA and TIMP3 siRNA. The data showed that co-silencing of P27 and TIMP3 did not have additive effect on PASMC proliferation (Fig. 5).

The regulation of miR-222 and its target genes under hypoxia

To reveal the physiological relevance of miR-222 for PASMC proliferation and eventually $\mathrm{PAH}$, experiments under hypoxic condition were performed. The data showed that miR222 was upregulated in PASMCs under hypoxic condition for 24 hours (Fig. 6A). Hypoxia 
Fig. 2. $P 27$ is a target gene of miR-222 in pulmonary artery smooth muscle cells (PASMCs). (A) miR222 negatively regulated the protein expression level of P27 in PASMCs. (B) P27 was efficiently downregulated at protein level in PASMCs transfected with P27 siRNA. (C-E) miR-222 mimic or P27 siRNA alone could promote PASMC proliferation, whereas they did not have additive effect as determined by EdU assay (C), Ki67 staining (D), and quantification of cell number (E). (F-H) P27 siRNA could attenuate the anti-proliferation effect of miR-222 inhibitor in PASMCs as determined by EdU assay (F), Ki67 staining $(G)$, and quantification of cell number $\mathrm{H}$. For immunofluo-

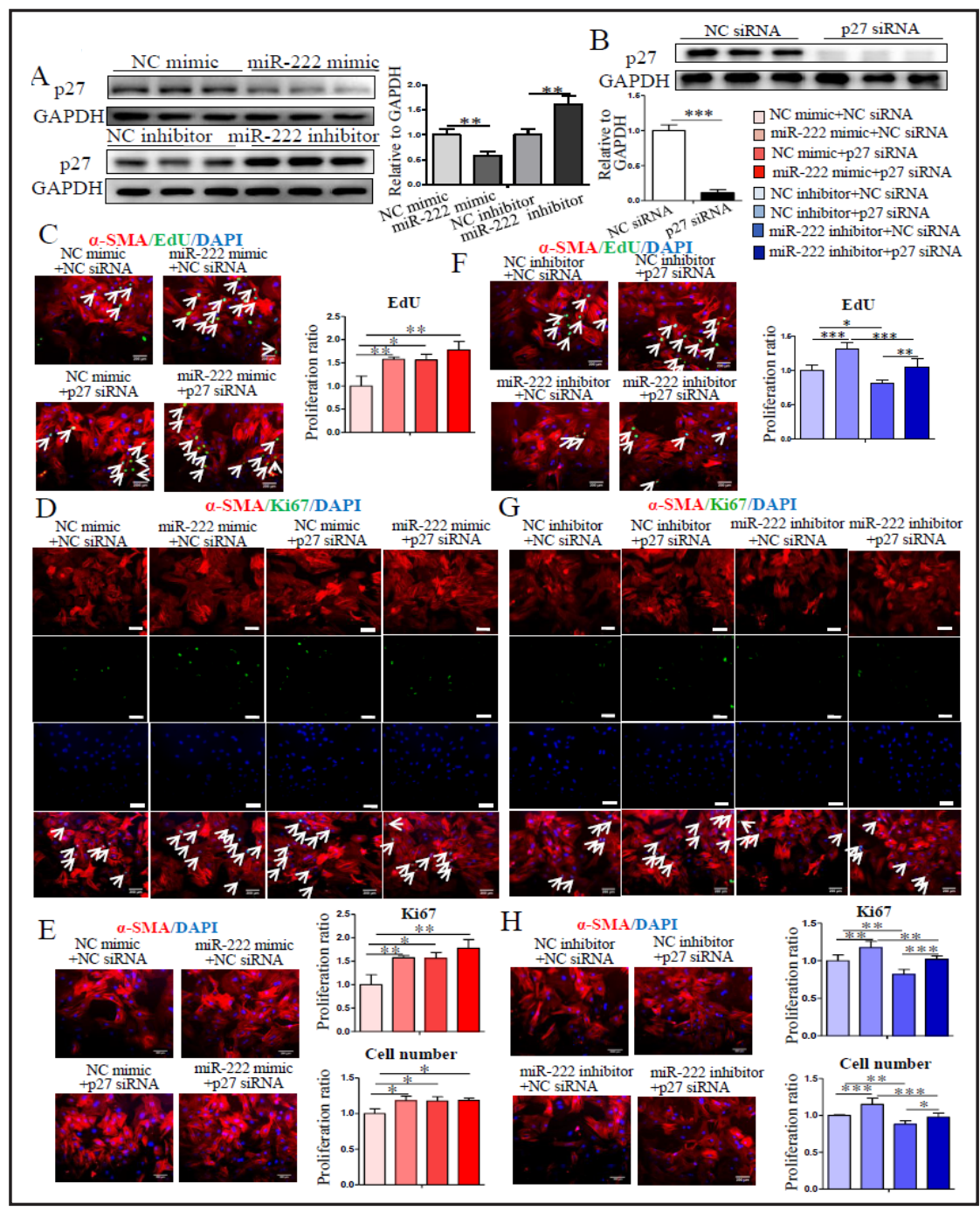
rescent stainings, nuclei were stained by DAPI. The arrows indicate EdU-positive or Ki67-positive PASMCs. Scale bar: $200 \mu$ m. $^{*}, P<0.05$; $^{* *}, P<0.01$; ${ }^{* * *}, P<0.001 . \mathrm{n}=6$ per group.

Fig. 3. TIMP3 is a direct target gene of miR-222. (A) Schematic representation of pmiR-RB-Report containing a fragment of the $3^{\prime}$ UTR of TIMP3 mRNA with the putative miR-222 binding sequences (wt) or without the putative miR222 binding sequences (mutant). (B) Luciferase assays confirmed TIMP3 as a direct target gene of miR-222. (C) miR-222 negatively regulated the protein expression

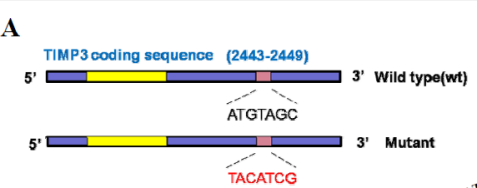

B

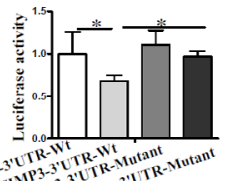

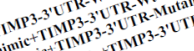

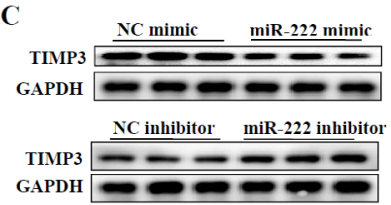

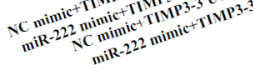

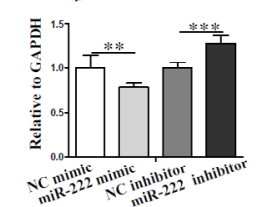

level of TIMP3 in pulmonary artery smooth muscle cells (PASMCs). ${ }^{*}, P<0.05$; $^{* *}, P<0.01$; $^{* *}, P<0.001 . \mathrm{n}$ $=6$ per group.

promoted PASMC proliferation which was attenuated by miR-222 inhibitor (Fig. 6B and C). In addition, P27 and TIMP3 were both downregulated in PASMCs under hypoxia, which could be reversed by miR-222 inhibitor (Fig. 6D). 
Fig. 4. TIMP3 mediates the effect of miR-222 in the regulation of pulmonary artery smooth muscle cell (PASMC) proliferation. (A) TIMP3 was efficiently downregulated at protein level in PASMCs transfected with TIMP3 siRNA. (B-D) miR-222 mimic or TIMP3 siRNA alone could promote PASMC proliferation, whereas they did not have additive effect as determined by EdU assay (B), Ki67 staining (C), and quantification of cell number (D). (E-G) TIMP3 siRNA could attenuate the anti-proliferation effect of miR-222 inhibitor in PASMCs as determined by EdU assay (E), Ki67 staining (F), and quantification of cell number (G). For immunofluorescent stainings, nuclei were stained by DAPI. The arrows indicate EdU-positive or Ki67positive PASMCs. Scale bar: $200 \mu \mathrm{m} .{ }^{*}, P<0.05$; **, $P<0.01$; ${ }^{* *}, P<0.001 . \mathrm{n}=$ 6 per group.
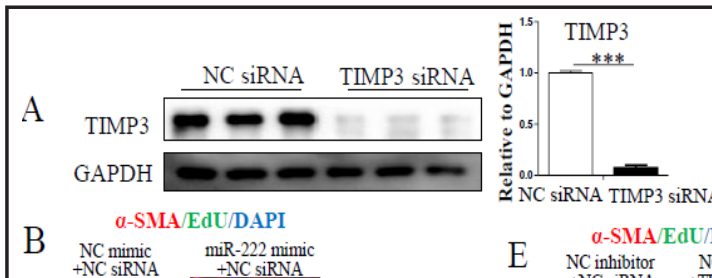

$\square \mathrm{NC}$ mimic $+\mathrm{NC}$ siRNA

miR-222 mimic $+N C$ siRNA

$\checkmark$ NC mimic+TIMP3 siRNA

miR-222 mimic+TIMP3 siRNA

$\square \mathrm{NC}$ inhibitor $+\mathrm{NC}$ siRNA

$\square$ NC inhibitor+TIMP3 siRNA

$\approx$ NC siRNA TIMP3 siRNA miR-222 inhibitor+NC siRNA a-SMA/EdU/DAPI

miR-222 inhibitor+TIMP3 siRNA

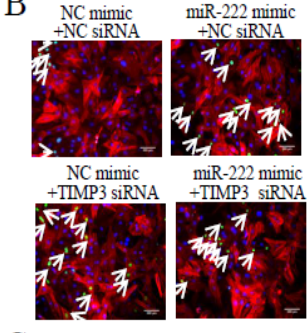

E NC inhibitor NC inhibitor
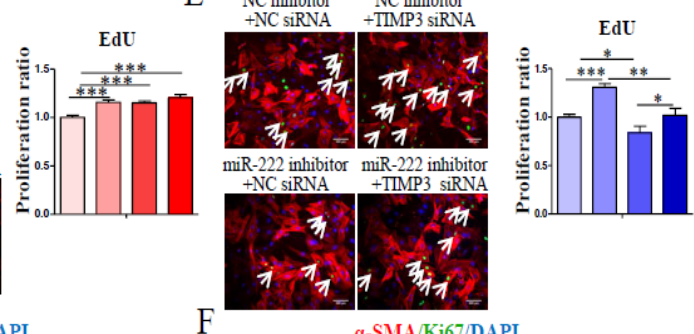

C

NC mimic
+ +NC siRNA $\begin{gathered}\text { miR-222 mimic } \\ \text { +NC siRNA }\end{gathered}$

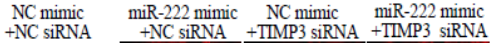

$\alpha$-SMA/Ki67/DAPI

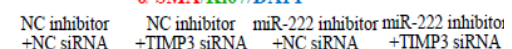

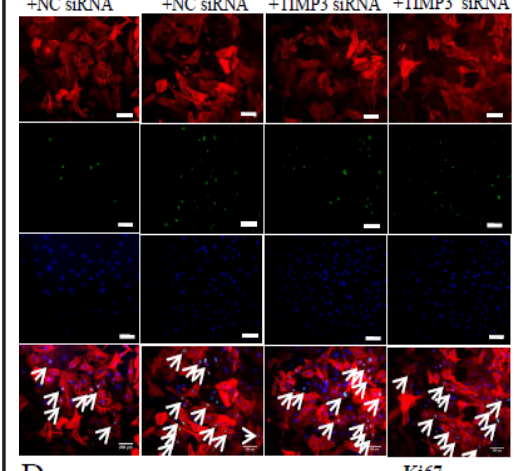

D $\alpha$-SMA/DAPI
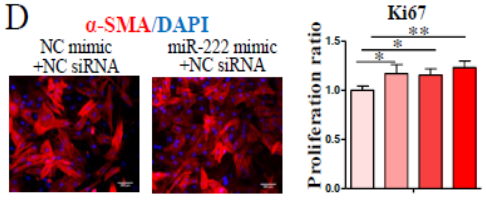

$\underset{+\mathrm{NCmimic}}{\mathrm{N} M \mathrm{MP} 3 \text { SiRNA }}$

miR-222 mimic miR-222 mimic
+TIMP3 siRNA

Cell number
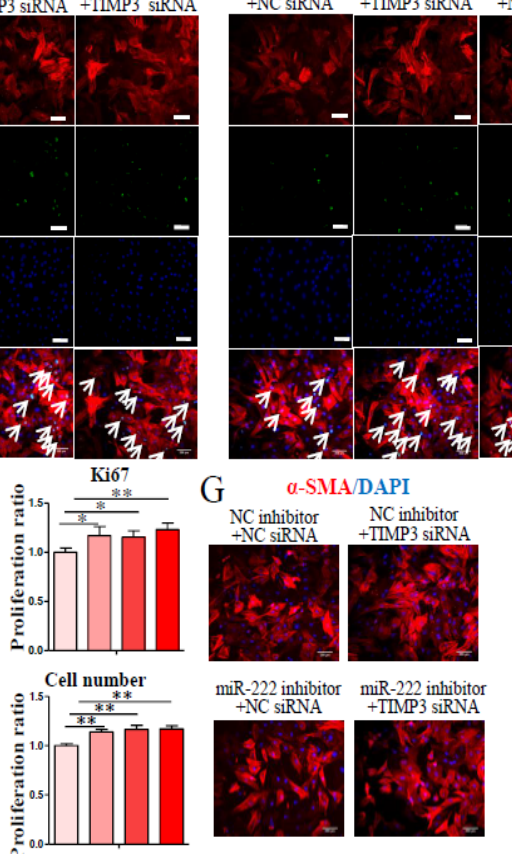

SMA/DAPI NC inhibitor

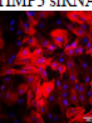

miR-222 inhibitor miR-222 inhibitor
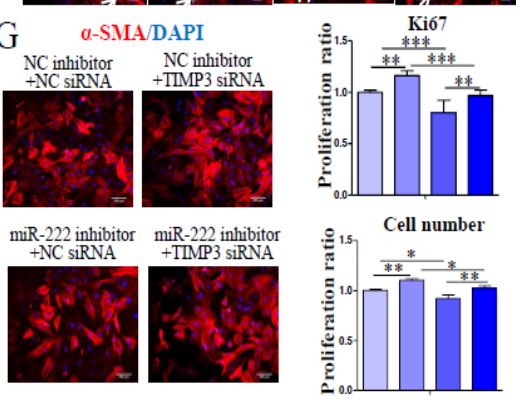

\section{Discussion}

It is well known that aberrant vascular smooth muscle cell (VSMC) proliferation plays an important role in the development of many proliferative cardiovascular diseases, such as atherosclerosis, vein graft failure, restenosis after angioplasty, as well as PAH [1, 3]. Inhibition of VSMC proliferation could lead to better prevention and treatment of these diseases.

Increasing evidence shows that miRNAs play pivotal roles in many biological activities, including VSMC proliferation. It has been reported that miR-222 could promote proliferation and migration, while inhibit apoptosis of aortic SMCs [31]. However, the role of miR-222 in PASMCs is unclear. In this study, we demonstrate that miR-222 has a pro-proliferation effect on SMCs derived from pulmonary artery as determined by PCNA expression, Ki-67 and EdU staining, and total cell number counting.

A single miRNA can target several target genes while a single gene can also be regulated by several miRNAs. P27 is a well-known target gene of miR-222 in several types of cells including VSMCs. As a member of the Cip/Kip family of cyclin-dependent kinase inhibitors, P27 has been found to negatively regulate cell proliferation including cancer cells and VSMCs [36]. In the present study, we found a negative regulation of P27 by miR-222 in PASMCs 
Fig. 5. Effect of simultaneous silence of P27 and TIMP3 on the proliferation of pulmonary artery smooth muscle cells (PASMCs) with miR-222 inhibition. Cotransfection of P27 siRNA and TIMP3 siRNA did not have additive effect on PASMC proliferation as determined by EdU assay (A), Ki67 staining (B), and quantification of cell number (C). For immunofluorescent stainings, nuclei were stained by DAPI. The arrows indicate EdU-positive or Ki67-positive PASMCs. Scale bar: $200 \mu \mathrm{m} .{ }^{*}, P<$ 0.05 ; **, $P<0.01$; ***,$P<$ $0.001 . \mathrm{n}=6$ per group.

Fig. 6. Inhibition of miR222 reduces hypoxiainduced proliferation of pulmonary artery smooth muscle cells (PASMCs). (A) miR-222 was upregulated in PASMCs under hypoxic condition for 24 hours. (B, C) miR-222 inhibitor reduced hypoxia-induced PASMC proliferation as determined by EdU assay (B) and Ki67 staining (C). (D) The TIMP3 and P27 protein expressions were down-regulated in PASMCs under hypoxic condition, which was reversed by miR-222 inhibition. For immunofluorescent stainings, nuclei were stained by DAPI. The arrows indicate EdU-positive or Ki67positive PASMCs. Scale bar: $200 \mu \mathrm{m} .{ }^{*}, P<0.05$; **, $P<0.01 ;{ }^{* * *}, P<0.001 . \mathrm{n}=$ 6 per group.
A

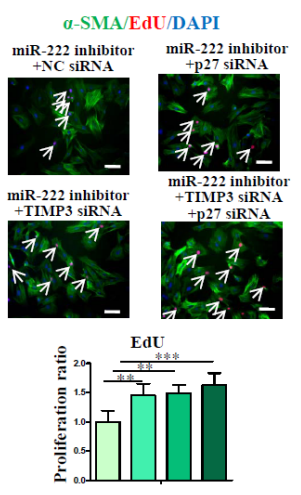

C

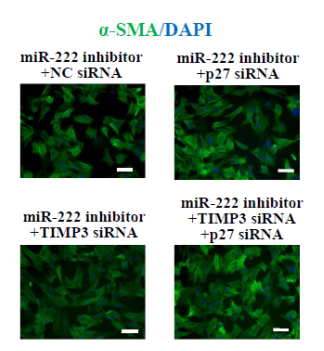

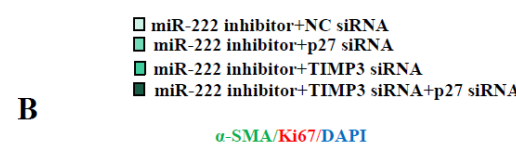

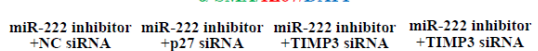
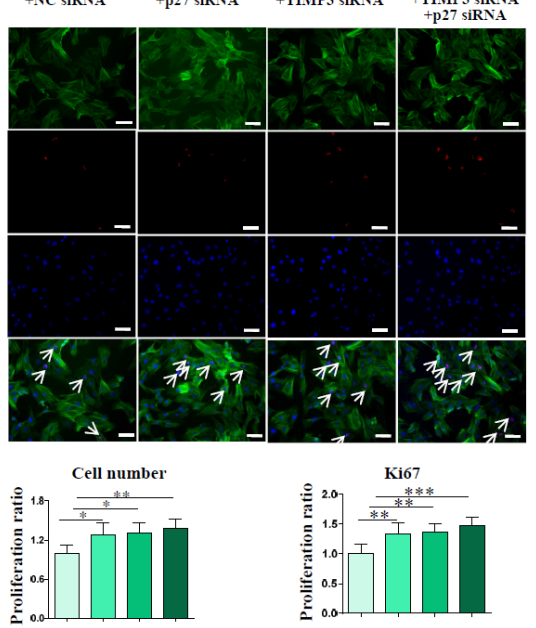

Ki67

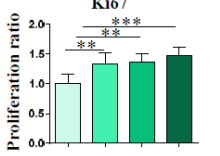

A

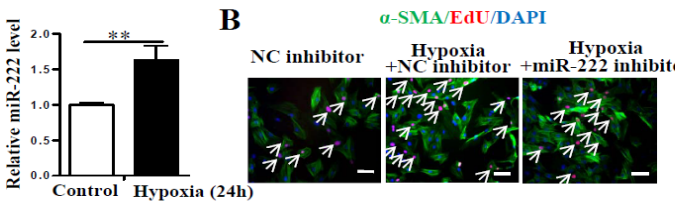

C

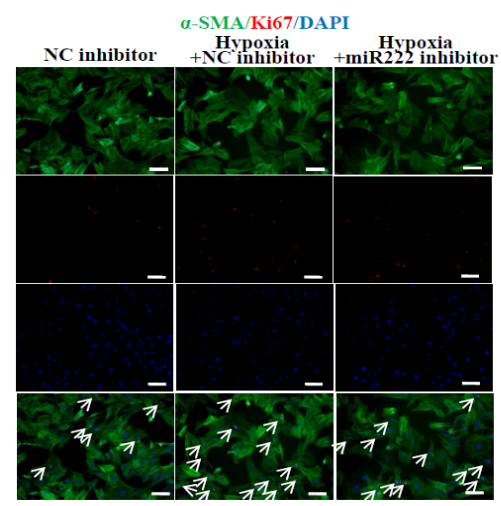

D

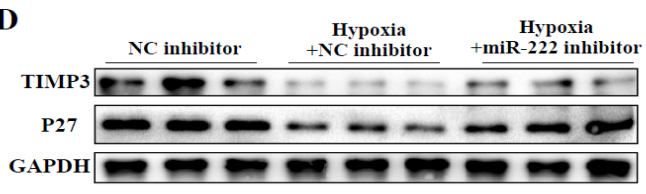

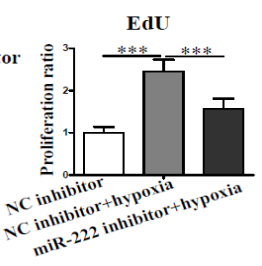

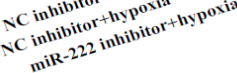

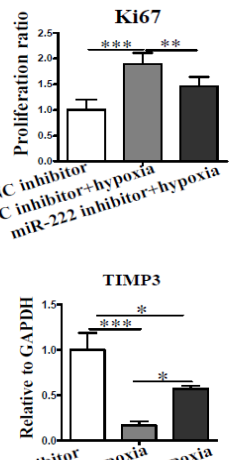

innibititer

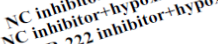

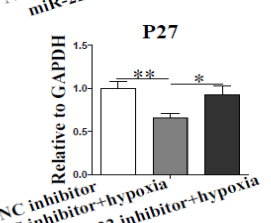


and P27 inhibition was responsible for the pro-proliferation effect of miR-222 in PASMCs. In addition, TIMP3 is a newly found target gene of miR-222 [28, 35]. As a member of the TIMP family, TIMP3 can regulate many physiological effects including cell proliferation, apoptosis, and migration through MMP-dependent or MMP-independent manner [34, 35]. It has been proved that miR-222 could induce neuronal apoptosis by targeting TIMP3 in vitro [34]. However, if TIMP3 is a target gene of miR-222 in PASMCs has not been determined. In the current study, we identified that TIMP3 was a target gene of miR-222 in PASMCs, as confirmed by luciferase assay and the negative regulation of TIMP3 by miR-222 in PASMCs. Importantly, co-transfection of miR-222 mimic and TIMP3 siRNA could not further enhance the proliferation of PASMCs while TIMP3 siRNA could attenuate the anti-proliferation effect of miR-222 inhibitor in PASMCs, indicating that TIMP3 inhibition is also responsible for the pro-proliferation effect of miR-222 in PASMCs. In addition, simultaneous knock-down of P27 and TIMP3 did not show an additive effect on PASMC proliferation, indicating that the two genes might be regulated and act in a similar way. Collectively, P27 and TIMP3 were identified as two target genes of miR-222 in regulating PASMC proliferation.

Chronic hypoxia is one of the major causes of pulmonary hypertension. Persistent hypoxia can result in endothelial dysfunction, uncontrolled cell proliferation, and pulmonary vascular remodeling [37]. As hypoxia is a common symptom of pulmonary hypertension which can promote the proliferation of PASMCs, we also examined whether miR-222 could regulate PASMC proliferation under hypoxic condition. We found an upregulation of miR222 in hypoxic PASMCs. More importantly, hypoxia-induced PASMC proliferation can be reduced by miR-222 inhibition. In addition, P27 and TIMP3 expressions were both downregulated in PASMCs under hypoxic condition and were reversed by miR-222 inhibitor. These data provide evidence indicating that miR-222 inhibition may exert beneficial effect in reducing PASMC proliferation in pulmonary hypertension related to hypoxic conditions such as chronic obstructive pulmonary disease and interstitial lung disease [38, 39].

In conclusion, miR-222 promotes PASMC proliferation at least partially through targeting P27 and TIMP3. Therefore, inhibition of miR-222 in PASMCs may be a potential therapy for PAH.

\section{Acknowledgements}

This work was supported by the grants from National Natural Science Foundation of China (91639101 and 81570362 to JJ Xiao, 81370332 and 81170201 to XL Li, 81400647 to Y Bei), Innovation Program of Shanghai Municipal Education Commission (2017-01-0700-09-E00042 to JJ Xiao), the grant from Science and Technology Commission of Shanghai Municipality (17010500100), the development fund for Shanghai talents (to JJ Xiao), the Priority Academic Program Development of Jiangsu Higher Education Institutions (PAPD20102013 to XL Li), and the development fund for Shanghai talents (to JJ Xiao). Dr XL $\mathrm{Li}$ is an Associate Fellow at the Collaborative Innovation Center for Cardiovascular Disease Translational Medicine.

\section{Disclosure Statement}

The authors declare there are no conflicts of interest.

\section{References}

1 McLaughlin VV: Looking to the future: A new decade of pulmonary arterial hypertension therapy. Eur Respir Rev 2011;20:262-269.

2 Fine NM, Chen L, Bastiansen PM, Frantz RP, Pellikka PA, Oh JK, Kane GC: Outcome prediction by quantitative right ventricular function assessment in 575 subjects evaluated for pulmonary hypertension. Circ Cardiovasc Imaging 2013;6:711-721. 


\section{Cellular Physiology Cell Physiol Biochem 2017;43:282-292 \begin{tabular}{c|c|c|c|} 
DOI: 10.1159/000480371 & C 2017 The Author(s). Published by S. Karger AG, Basel \\
www.karger.com/cpb
\end{tabular}

- Morrell NW, Adnot S, Archer SL, Dupuis J, Jones PL, MacLean MR, McMurtry IF, Stenmark KR, Thistlethwaite PA, Weissmann N, Yuan JX, Weir EK: Cellular and molecular basis of pulmonary arterial hypertension. J Am Coll Cardiol 2009;54:S20-31.

4 Ambros V: The functions of animal micrornas. Nature 2004;431:350-355.

5 Farh KK, Grimson A, Jan C, Lewis BP, Johnston WK, Lim LP, Burge CB, Bartel DP: The widespread impact of mammalian micrornas on mrna repression and evolution. Science 2005;310:1817-1821.

6 Ambros V: Microrna pathways in flies and worms: Growth, death, fat, stress, and timing. Cell 2003;113:673676.

7 Bartel DP: Micrornas: Genomics, biogenesis, mechanism, and function. Cell 2004;116:281-297.

-8 Zheng Z, Li X, Zhu Y, Gu W, Xie X, Jiang J: Prognostic significance of mirna in patients with diffuse large b-cell lymphoma: A meta-analysis. Cell Physiol Biochem 2016;39:1891-1904.

-9 Caruso P, Dempsie Y, Stevens HC, McDonald RA, Long L, Lu R, White K, Mair KM, McClure JD, Southwood M, Upton P, Xin M, van Rooij E, Olson EN, Morrell NW, MacLean MR, Baker AH: A role for mir-145 in pulmonary arterial hypertension: Evidence from mouse models and patient samples. Circ Res 2012;111:290-300.

10 Gupta S, Li L: Modulation of mirnas in pulmonary hypertension. Int J Hypertens 2015;2015:169069.

11 Zhou S, Li M, Zeng D, Xu X, Fei L, Zhu Q, Zhang Y, Wang R: A single nucleotide polymorphism in 3' untranslated region of epithelial growth factor receptor confers risk for pulmonary hypertension in chronic obstructive pulmonary disease. Cell Physiol Biochem 2015;36:166-178.

12 Deng L, Blanco FJ, Stevens H, Lu R, Caudrillier A, McBride M, McClure JD, Grant J, Thomas M, Frid M, Stenmark K, White K, Seto AG, Morrell NW, Bradshaw AC, MacLean MR, Baker AH: Microrna-143 activation regulates smooth muscle and endothelial cell crosstalk in pulmonary arterial hypertension. Circ Res 2015;117:870-883.

13 Pullamsetti SS, Doebele C, Fischer A, Savai R, Kojonazarov B, Dahal BK, Ghofrani HA, Weissmann N, Grimminger F, Bonauer A, Seeger W, Zeiher AM, Dimmeler S, Schermuly RT: Inhibition of microrna-17 improves lung and heart function in experimental pulmonary hypertension. Am J Respir Crit Care Med 2012;185:409-419.

14 Parikh VN, Jin RC, Rabello S, Gulbahce N, White K, Hale A, Cottrill KA, Shaik RS, Waxman AB, Zhang YY, Maron BA, Hartner JC, Fujiwara Y, Orkin SH, Haley KJ, Barabasi AL, Loscalzo J, Chan SY: Microrna-21 integrates pathogenic signaling to control pulmonary hypertension: Results of a network bioinformatics approach. Circulation 2012;125:1520-1532.

15 Guo L, Qiu Z, Wei L, Yu X, Gao X, Jiang S, Tian H, Jiang C, Zhu D: The microrna-328 regulates hypoxic pulmonary hypertension by targeting at insulin growth factor 1 receptor and l-type calcium channelalpha1c. Hypertension 2012;59:1006-1013.

16 Boucherat O, Potus F, Bonnet S: Microrna and pulmonary hypertension. Adv Exp Med Biol 2015;888:237252.

17 Yue J, Guan J, Wang X, Zhang L, Yang Z, Ao Q, Deng Y, Zhu P, Wang G: Microrna-206 is involved in hypoxia-induced pulmonary hypertension through targeting of the hif-1alpha/fhl-1 pathway. Lab Invest 2013;93:748-759.

18 Bertero T, Lu Y, Annis S, Hale A, Bhat B, Saggar R, Saggar R, Wallace WD, Ross DJ, Vargas SO, Graham BB, Kumar R, Black SM, Fratz S, Fineman JR, West JD, Haley KJ, Waxman AB, Chau BN, Cottrill KA, Chan SY: Systems-level regulation of microrna networks by mir-130/301 promotes pulmonary hypertension. J Clin Invest 2014;124:3514-3528.

19 Bertero T, Cottrill K, Krauszman A, Lu Y, Annis S, Hale A, Bhat B, Waxman AB, Chau BN, Kuebler WM, Chan SY: The microrna-130/301 family controls vasoconstriction in pulmonary hypertension. J Biol Chem 2015;290:2069-2085.

20 Chen T, Zhou G, Zhou Q, Tang H, Ibe JC, Cheng H, Gou D, Chen J, Yuan JX, Raj JU: Loss of microrna-17 approximately 92 in smooth muscle cells attenuates experimental pulmonary hypertension via induction of pdz and lim domain 5. Am J Respir Crit Care Med 2015;191:678-692.

-21 Huber LC, Ulrich S, Leuenberger C, Gassmann M, Vogel J, von Blotzheim LG, Speich R, Kohler M, Brock M: Featured article: Microrna-125a in pulmonary hypertension: Regulator of a proliferative phenotype of endothelial cells. Exp Biol Med (Maywood) 2015;240:1580-1589.

22 Xing Y, Zheng X, Li G, Liao L, Cao W, Xing H, Shen T, Sun L, Yang B, Zhu D: Microrna-30c contributes to the development of hypoxia pulmonary hypertension by inhibiting platelet-derived growth factor receptor beta expression. Int J Biochem Cell Biol 2015;64:155-166. 


\section{Cellular Physiology Cell Physiol Biochem 2017;43:282-292 \begin{tabular}{ll|l} 
DOI: 10.1159/000480371 30, 2017 & $\begin{array}{l}\text { O } 2017 \text { The Author(s). Published by S. Karger AG, Basel } \\
\text { www.karger.com/cpb }\end{array}$
\end{tabular}

-23 McLendon JM, Joshi SR, Sparks J, Matar M, Fewell JG, Abe K, Oka M, McMurtry IF, Gerthoffer WT: Lipid nanoparticle delivery of a microrna-145 inhibitor improves experimental pulmonary hypertension. J Control Release 2015;210:67-75.

24 Potus F, Ruffenach G, Dahou A, Thebault C, Breuils-Bonnet S, Tremblay E, Nadeau V, Paradis R, Graydon C, Wong R, Johnson I, Paulin R, Lajoie AC, Perron J, Charbonneau E, Joubert P, Pibarot P, Michelakis ED, Provencher S, Bonnet S: Downregulation of microrna-126 contributes to the failing right ventricle in pulmonary arterial hypertension. Circulation 2015;132:932-943.

-25 Liu X, Xiao J, Zhu H, Wei X, Platt C, Damilano F, Xiao C, Bezzerides V, Bostrom P, Che L, Zhang C, Spiegelman $\mathrm{BM}$, Rosenzweig A: Mir-222 is necessary for exercise-induced cardiac growth and protects against pathological cardiac remodeling. Cell Metab 2015;21:584-595.

-26 Su M, Chen Z, Wang C, Song L, Zou Y, Zhang L, Hui R, Wang J: Cardiac-specific overexpression of mir-222 induces heart failure and inhibits autophagy in mice. Cell Physiol Biochem 2016;39:1503-1511.

27 Li Y, Liang C, Ma H, Zhao Q, Lu Y, Xiang Z, Li L, Qin J, Chen Y, Cho WC, Pestell RG, Liang L, Yu Z: Mir221/222 promotes s-phase entry and cellular migration in control of basal-like breast cancer. Molecules 2014;19:7122-7137.

28 Zhang C, Zhang J, Hao J, Shi Z, Wang Y, Han L, Yu S, You Y, Jiang T, Wang J, Liu M, Pu P, Kang C: High level of mir-221/222 confers increased cell invasion and poor prognosis in glioma. J Transl Med 2012;10:119.

29 Bazan HA, Hatfield SA, O'Malley CB, Brooks AJ, Lightell D, Jr., Woods TC: Acute loss of mir-221 and mir-222 in the atherosclerotic plaque shoulder accompanies plaque rupture. Stroke 2015;46:3285-3287.

-30 Liu X, Cheng Y, Zhang S, Lin Y, Yang J, Zhang C: A necessary role of mir-221 and mir-222 in vascular smooth muscle cell proliferation and neointimal hyperplasia. Circ Res 2009;104:476-487.

31 Liu X, Cheng Y, Yang J, Xu L, Zhang C: Cell-specific effects of mir-221/222 in vessels: Molecular mechanism and therapeutic application. J Mol Cell Cardiol 2012;52:245-255.

-32 Lambeth LS, Yao Y, Smith LP, Zhao Y, Nair V: Micrornas 221 and 222 target p27kip1 in marek's disease virus-transformed tumour cell line msb-1. J Gen Virol 2009;90:1164-1171.

33 Zhong C, Ding S, Xu Y, Huang H: Microrna-222 promotes human non-small cell lung cancer h460 growth by targeting p27. Int J Clin Exp Med 2015;8:5534-5540.

34 Zhou S, Zhang S, Wang Y, Yi S, Zhao L, Tang X, Yu B, Gu X, Ding F: Mir-21 and mir-222 inhibit apoptosis of adult dorsal root ganglion neurons by repressing timp3 following sciatic nerve injury. Neurosci Lett 2015;586:43-49.

35 Lu Y, Roy S, Nuovo G, Ramaswamy B, Miller T, Shapiro C, Jacob ST, Majumder S: Anti-microrna-222 (antimir-222) and -181b suppress growth of tamoxifen-resistant xenografts in mouse by targeting timp3 protein and modulating mitogenic signal. J Biol Chem 2011;286:42292-42302.

-36 Toyoshima H, Hunter T: P27, a novel inhibitor of g1 cyclin-cdk protein kinase activity, is related to p21. Cell 1994;78:67-74.

-37 Duong-Quy S, Bei Y, Liu Z, Dinh-Xuan AT: Role of rho-kinase and its inhibitors in pulmonary hypertension. Pharmacol Ther 2013;137:352-364.

-38 Zhang WF, Zhu TT, Xiong YW, Xiong AZ, Ge XY, Hu CP, Zhang Z: Negative feedback regulation between microrna let-7g and lox-1 mediated hypoxia-induced pasmcs proliferation. Biochem Biophys Res Commun 2017;488:655-663.

39 Bei Y, Hua-Huy T, Duong-Quy S, Nguyen VH, Chen W, Nicco C, Batteux F, Dinh-Xuan AT: Long-term treatment with fasudil improves bleomycin-induced pulmonary fibrosis and pulmonary hypertension via inhibition of smad2/3 phosphorylation. Pulm Pharmacol Ther 2013;26:635-643. 